\title{
Optimization of path availability of span-restorable optical networks
}

\author{
Ling Zhou*, Marcel Held \\ Swiss Federal Laboratories for Materials Testing and Research (EMPA), CH-8600 Dübendorf, \\ Switzerland, *ling.zhou@empa.ch; phone +41 4482348 34; fax +41 4482340 54; www.empa.ch
}

\begin{abstract}
High availability tops the list of features desired for building a network. Various factors influencing path availability of optical networks with span restoration are investigated from the network design point of view. Network redundancy, number of backup routes, and dual span failure restorability can have important effects on path availability. A formulation to improve average path availability of a network by maximizing dual failure restorability is developed. We also introduce a practical method to optimize spare capacity and path availability of a network at the same time. A case study analyzes how much average dual-failure restorability can be improved in a long haul network with span restoration. Conclusions deduced from the computational and analytical results can help network planners to design a network with high performance and optimized cost and availability.
\end{abstract}

Keywords: Path Unavailability, Dual failure restorability, Integer Linear Programming, Optimization, Span Restoration.

\section{INTRODUCTION}

In optical transport networks, survivability is an essential requirement. The availability of a specific service path is of interest for network planners, service providers, and customers. High path availability can ensure credible availability guarantees in a service level agreement. Service providers can thus attract more customers and have big potential to increase the revenue. Path availability can be improved usually by installing reliable equipment and operation software, and by reducing mean time to repair as a result of online monitoring of optical parameters. In this paper, we try to find a solution to improve the path availability from the standpoint of network design, such as capacity allocation and network redundancy, when all the physical equipment for a network has been determined. Network planners can, in effect, make wise design choices for path availability improvement early in the development cycle and later when the additional investment is planned.

A network is commonly designed to be $100 \%$ restorable to any single span failure. However, node failures and multiple failures are not taken into account. Once multiple failures occur, a network may not be fully restorable anymore. According to the concept of "most likely paths to failure" [1], the simplest combinations of elemental failures that lead to an outage state in any system with redundancy will dominate the overall availability. Evaluating and summing the probabilities of those dominant classes of failure combinations gives a good approximation to the actual failure probability, i.e. the system unavailability. Dual-span failures are the next dominant failure modes in a network designed with full restorability to single span failures. Dual-span failures can occur simultaneously or within some interval. The latter situation can be depicted as follows: after the first span failure occurs, the second span failure appears before the repair to the first failure is finished.

\subsection{State of the art}

Some work has been done to improve the availability of networks by different methods. A method for the assessment of end-to-end path availability in span-restorable mesh networks was proposed [2] under the assumption that all spans have the same physical unavailability and no quantitative value for path availability was presented. This work also compared dual-failure restorability levels of different networks plotted against their corresponding network redundancies and concluded that the availability depends more on the individual network and the demand pattern than on the simple network redundancy. One striking finding in [3] was that limited resource sharing restricted flexibility in finding capacity after failures and accordingly limited the capability of reprovisioning schemes in improving the network restorability. The work in [4] studied the problem of improving service availability under dual near-simultaneous failures and proposed a re-provisioning algorithm to improve the robustness of optical mesh networks. The tradeoffs between

\footnotetext{
Reliability of Optical Fiber Components, Devices, Systems, and Networks III, edited by Hans G. Limberger, M. John Matthewson,

Proc. of SPIE Vol. 6193, 619312, (2006) - 0277-786X/06/\$15 - doi: 10.1117/12.662095
}

Proc. of SPIE Vol. $6193619312-1$ 
robustness and efficiency were examined in [5] and theoretical models were developed to find useful performance bounds. The work presented in [6] further considered the behaviour of networks that supported multiple types of protection schemes and examined by simulations a network that supported both $1+1$ protection and dynamic recovery to determine the optimal mixture of the two schemes. The performance of the sub-graph routing strategy and the backup multiplexing scheme for tolerating a second link failure in a network was evaluated and compared in [7]. A formulation about maximizing the achievable dual-failure restorability level with a given total spare capacity investment was given in [8] for the scenario of two simultaneous span failures.

\subsection{Outline}

In Section 2 we introduce our concept of path unavailability suitable to span restoration and present the methodology for its calculation. We briefly analyze the relevant factors for path unavailability from the standpoint of network design. An integer linear programming (ILP) formulation with the objective to maximize dual span failure restorability $\left(R_{2}\right)$ is proposed also in this section and addresses the case of non-simultaneous dual-span failures. A practical application about this formulation is discussed. In Section 3, we make case studies on five networks and implement two experiments. One experiment aims to check the enhancement of $R_{2}$ from that ILP formulation. The other tries to find the affecting factors for path unavailability. Finally, in Section 4 the conclusions are given.

\section{METHODOLOGY}

\subsection{Path Unavailability}

In optical transport networks, present survivability strategies have the common objective to protect a network against one single span failure. Dual span failures are then the next key-factor determining network restorability and availability. Availability calculation for a specific path is straightforward for dedicated automatic protection switching (1+1 APS) and can be obtained from an analytical calculation or numerical simulation of the derived reliability block diagram (RBD). Therefore, the path unavailability of 1+1 APS is just the connection unavailability. Availability analysis based on reliability block diagrams is, however, not suitable for the dual failure case of mesh-restorable networks, because the network redundancy is obtained from shared spare capacity which is allocated to physically distributed spans and routes. It is hard to explicitly represent each item of a path in such networks with RBDs [1,9]. The following concept of availability analysis can facilitate the assessment of the path availability in networks.

In the availability analysis of optical networks, unavailability quantities are often applied to simplify many expressions. It is known that unavailabilities add up for independent elements in series. Therefore, the unavailability of a path $U_{\text {path }}$ in an unprotected network is the sum of the physical unavailability $U_{i}^{\text {phy }}$ of each span on the path.

$$
U_{p a t h} \approx \sum_{i=1}^{n} U_{i}^{p h y}
$$

A path in a span-restorable network has a similar serial structure. We use $U_{i}^{*}$ to express the equivalent unavailability of span $i$ considering the restoration effect instead of $U_{i}^{\text {phy }}$ in Eq. (1). The path unavailability in such a network is then $[1,9]$

$$
U_{\text {path }} \approx \sum_{i=1}^{n} U_{i}^{*}
$$

In a span-restorable network designed with full restorability to single-span failures, dual span failures occurring independently are assumed to dominate the system unavailability. The probability of a dual-span failure $(i, j)$ is the product of physical unavailabilities of two spans, i.e. $U_{i}^{p h y} \times U_{j}^{p h y}$ [1]. The two failed spans $i$ and $j$ thus contribute $U_{i}^{p h y} \times U_{j}^{p h y}$ to the unavailability $U_{i}^{*}$ of span $i$, which is on the working path between two considered nodes, if the traffic on span $i$ cannot be fully restored due to the dual-span failure.

Let $N_{i}$ represent the un-restored capacity of span $i$ caused by dual span failures. The general idea of restorationaware path unavailability for span-restorable networks is that any dual failure combination leading to $N_{i}>0$ will give its 
contribution to the unavailability of the considered span irrespective of the sequence of failures. Therefore we can express $U_{i}^{*}$ as follows:

$$
U_{i}^{*}=U_{i}^{p h y} \times \sum_{j \in S \mid j \neq i} c_{j} U_{j}^{p h y}
$$

where $c_{j}=0,0.5,1 . S$ denotes the set of spans in the network. If the failed span pair $(i, j)$ does not lead to un-restored capacity, it does not give a contribution to the unavailability $U_{i}^{*}$ of $\operatorname{span} i$ and thus $c_{j}=0$. If $\operatorname{span} j$ is on the working path to which span $i$ belongs, $c_{j}=0.5$ may be considered to avoid double counting in the computation of path unavailability when the failed span pair $(i, j)$ causing $N_{i}>0$ gives its contribution to both $U_{i}^{*}$ and $U_{j}^{*}$. Except for the two above cases, the failed span pair $(i, j)$, which causes $N_{i}>0$, gives its contribution to $U_{i}^{*}$ with $c_{j}=1$.

After having the unavailability of a path in a span-restorable network, we can define the traffic loss of a path accordingly, i.e. restoration-aware path traffic loss (RPTL) (Gb/year). We start to define $R P T L_{i}^{*}$, the restoration-aware traffic loss of span $i$ :

$$
\operatorname{RPTL}_{i}^{*}=U_{i}^{p h y} \times \sum_{j \in S \mid j \neq i} c_{j} U_{j}^{p h y} N_{i}
$$

Considering that a dual-span failure can cause complete or partial traffic loss on the considered span in a span-restorable network, a minor modification can be made for Eq. (4) when the restoration details are not known.

$$
R P T L_{i}^{*}=U_{i}^{p h y} \times \sum_{j \in S \mid j \neq i}\left(c_{j} U_{j}^{p h y} N_{i} \frac{N_{i}}{w_{i}}\right)
$$

where $w_{i}$ is the working capacity of span $i$. The capacity fraction $N_{i} / w_{i}$ adjusts the probability $U_{i}^{p h y} \times U_{j}^{p h y}$.

The traffic loss of a service path between nodes $s$ and $t$ is

$$
R P T L_{s, t}=\sum_{i \in S \mid i \in W} R P T L_{i}^{*}
$$

where $W$ denotes the set of spans on the working path between nodes $s$ and $t$.

Then the average value for RPTL of all service paths can be called average restoration-aware path traffic loss (ARPTL) (Gb/year).

$$
A R P T L=\frac{1}{|N|(|N|-1)} \cdot \sum_{(s, t) \in N^{2} \mid s \neq t} R P T L_{s, t}
$$

where $N$ denotes the set of nodes in the network.

The physical unavailability $U_{i}^{p h y}$ of a span is [10]

$$
U_{i}^{p h y}=\frac{M T T R}{M T T F+M T T R}=\frac{\lambda_{S}}{\lambda_{S}+\mu} \approx \lambda_{S} \cdot M T T R
$$

where $M T T F=1 / \lambda_{S}$ is the mean time to failure and $M T T R=1 / \mu$ is the mean time to repair. $\lambda_{S}$ and $\mu$ are the failure rate and the repair rate of the span, respectively. For many practical cases of interest, MTTF $>>$ MTTR and $\lambda_{S}<<\mu$.

The failure rate of a span $\lambda_{S}$ is its length $L$ multiplied by the sum of the cable failure rate per kilometer $\lambda_{\text {cable }}$ and the optical amplifier failure rate per kilometer $\lambda_{O A}[11]$ :

$$
\lambda_{S}=L \cdot\left(\lambda_{\text {cable }}+C_{O A} \cdot \lambda_{O A}\right)
$$

where $C_{O A}$ is an optical amplifier spacing constant per kilometer.

Before the restoration details of a network are known, the above concept of path unavailability can only be an estimate of the unavailability of a service path, because all the traffic carried on a span is considered including the connection demand. Certainly, the concept can be extended to connection unavailability if detailed restoration routing is provided. 


\subsection{Parameter analysis of path unavailability}

After having a complete picture about the computation of path unavailability, we are able to analyse which parameters can affect the path unavailability systematically. From Eq. (8), $\lambda_{S}$ and MTTR both affect the physical unavailability of a span. The average repair time can be shortened by optical signal monitoring $[12,13]$ leading to shorter time for failure localization and correction. To reduce the failure rate of a span, more reliable optical cables and optical amplifiers (i.e. reducing $\lambda_{\text {cable }}$ and $\lambda_{O A}$ according to Eq. (9)) have to be used. The sensitivity analysis of path availability by varying these basic factors was presented in [11, 14]. According to Eq. (4) or (5), the path unavailability and the related traffic loss depend - in addition to the physical unavailabilities - on the un-restored capacity $N_{i}$ and the factor $c_{j}$. In other words, the average network dual failure restorability $R_{2}$, which definition is in [9], has to be improved as much as possible to decrease the units of any un-restorable capacity and the amount of $c_{j}$. This problem deals with an optimal network design and a total investment. A maximum $R_{2}$ design with a given total cost is given in the next section. The relationship among average path unavailability, capacity allocation, and network redundancy is shown in Section 3 by the case studies.

\subsection{Maximum dual failure restorability under static preplans}

The ILP formulation with the objective of obtaining dual failure maximum restorability (DFMR) in $[8,9]$ is suitable for the scenario of simultaneous dual-span failures and does not consider the restoration sequence of two failed spans. However, the other non-simultaneous dual-failure scenario can happen usually. Our following ILP formulation with the same objective, named single-failure pre-plan (SFPP), also addresses the latter scenario, except for the first scenario, in a static model of single span failures and indicates the restoration sequence explicitly.

$$
\text { SFPP: } \quad \text { Minimize } \sum_{(i, j) \in S^{2} \mid i \neq j} N(i, j)
$$

Subject to:

- Ensure full single failure restorability $\left(R_{I}=1\right)$ :

$$
\sum_{p \in P_{i}} f_{i}^{p}=w_{i}, \quad \forall i \in S
$$

- Un-restored working capacity:

$$
N(i, j)=w_{i}+w_{j}-\left[\sum_{p \in P_{i}} f_{i}^{p} \cdot\left(1-\delta_{i, j}^{p}\right)+\sum_{p \in P_{j}} f_{j, i}^{p}\right], \quad \forall(i, j) \in S^{2} \mid i \neq j
$$

- The restoration flow of the second failure cannot exceed the pre-planned flow for the single-failure scenario:

$$
f_{j, i}^{p} \leq f_{j}^{p} \cdot\left(1-\delta_{j, i}^{p}\right), \quad \forall(j, i) \in S^{2} \mid j \neq i, \forall p \in P_{j}
$$

- Basic spare capacity necessary to ensure $R_{l}=1$ :

$$
\sum_{p \in P_{i}} \delta_{i, k}^{p} \cdot f_{i}^{p} \leq s_{k} \quad \forall(i, k) \in S^{2} \mid i \neq k
$$

- $\quad$ Additional spare capacity necessary to enhance $R_{2}$ :

$$
\sum_{p \in P_{i}} f_{i}^{p} \cdot \delta_{i, k}^{p} \cdot\left(1-\delta_{i, j}^{p}\right)+\sum_{p \in P_{j}} f_{j, i}^{p} \cdot \delta_{j, k}^{p} \leq s_{k}, \quad \forall(i, j, k) \in S^{3} \mid i \neq j, i \neq k, j \neq k
$$

- Budget restriction:

$$
\sum_{k \in S} c_{k} \cdot s_{k} \leq B
$$

where

$i, j, k$ are the specific spans. $i$ denotes the first failed span and $j$ the second failed span. $k$ denotes a span which is neither span $i$ nor $\operatorname{span} j$.

$S$ is the set of spans in the network;

$P_{i}$ is the set of eligible candidate routes for the restoration of span $i$;

$c_{i}$ is the cost of each unit of capacity on span $i$ and can be the length of the span (input parameter);

$\delta_{i, k}^{p}$ is 1 if the $p^{t h}$ restoration route of span $i$ crosses span $k$, and 0 otherwise. The other expressions like $\delta_{j, k}^{p}, \delta_{i, j}^{p}$ and $\delta_{j, i}^{p}$ have the similar meaning when the corresponding notations are applied; 
$B$ is a budget limit for the total spare capacity cost (input parameter);

$w_{i}$ is the number of working capacity units on span $i$ (input parameter);

$s_{i}$ is the number of spare capacity units placed on span $i$ (non-negative integer variable);

$f_{i}^{p}$ is the restoration flow assigned to the $p^{\text {th }}$ restoration route of span $i$ as a single isolated-span failure scenario (non-negative integer variable);

$f_{j, i}^{p}$ is the restoration flow assigned to the $p^{\text {th }}$ restoration route of $\operatorname{span} j$ when span $i$ is also failed. This variable is always used to restore the second failure in this formulation (non-negative integer variable);

$N(i, j)$ is the number of non-restored working units under dual failure of spans $(i, j)$. The expression $(i, j)$ reflects the sequence of the two failed spans (non-negative integer variable).

Eq. (10) is the basic condition for span restoration to ensure full restorability to single span failures. Eq. (11) collects all the un-restored working capacity due to the failed spans $(i, j)$. The different definitions of $f_{i}^{p}$ and $f_{j, i}^{p}$ imply the restoration sequence, i.e. $f_{i}^{p}$ is responsible to restore the first failed span and $f_{j, i}^{p}$ is responsible for the second failed span. $f_{i}^{p}$ does not contribute its flow when span $j$ is on the $p^{\text {th }}$ restoration route of span i, i.e. $\delta_{i, j}^{p}=1$. Eq. (12) restricts the maximum value which $f_{j, i}^{p}$ can take. This formulation deals with the static pre-planned model of span restoration, in a way that the two span failures are restored according to their predetermined plans (including pre-planned routes and their corresponding flows) as if each failure were an isolated failure. Therefore the restoration flow for the second failure cannot exceed the pre-planned flow for the single-failure scenario. If span $i$ is a part of the restoration route of $\operatorname{span} j$, i.e. $\delta_{j, i}^{p}=1, f_{j, i}^{p}$ will be zero. Eq. (13) is another basic condition for span restoration to ensure $R_{l}=1$. Eq. (14) permits additional spare capacity for the dual failure scenario to be assigned optimally with the budget limit given in Eq. (15). Again, the restoration sequence for two failed spans is reflected in Eq. (14) and can directly guide network operators to make an optimal restoration under dual failure situations.

\subsection{Practical methods to achieve maximum $\boldsymbol{R}_{2}$}

Intuitionally, a direct and economical application to enhance $R_{2}$ can be implemented by setting the budget restriction with the minimum cost for the investigated network. First we apply basic spare capacity allocation (basic SCA) formulation [15] of span restoration to obtain the minimum necessary spare capacity with full restorability to single span failures. Note that basic SCA only provides minimum spare capacity of the network. There is still some space to enhance $R_{2}$ by purely rerouting the traffic. Then the result can be used as the input parameter $B$ in the above SFPP formulation. Finally we get the optimal result from SFPP with a new assignment of the restoration routes and an enhanced dual-failure restorability. This similar method applied with DFMR was described in [9] too.

If a big budget is allowed, an appropriate investment can be set accordingly. In Section 3, we will see that more spare capacity can achieve higher dual failure restorability, certainly before $R_{2}=1$ is reached.

\section{CASE STUDY}

Table 1: Test Network Characteristics

\begin{tabular}{|l|c|c|c|c|c|c|}
\hline Network & $\begin{array}{c}\# \\
\text { nodes }\end{array}$ & $\begin{array}{c}\# \\
\text { spans }\end{array}$ & $\begin{array}{c}\text { Average } \\
\text { nodal degree }\end{array}$ & $\begin{array}{c}\text { Degree-2 } \\
\text { Nodes }\end{array}$ & $\begin{array}{c}\text { Total length of } \\
\text { all spans (km) }\end{array}$ & $\begin{array}{c}\text { Average length } \\
\text { of a span (km) }\end{array}$ \\
\hline US net & 19 & 28 & 2.95 & 6 & 26066 & 930.9 \\
\hline n11s17 & 11 & 17 & 3.09 & 4 & 2490 & 146.5 \\
\hline n9s15 & 9 & 15 & 3.33 & 2 & 2755 & 183.7 \\
\hline n11s20 & 11 & 20 & 3.64 & 3 & 3093 & 154.7 \\
\hline n8s17 & 8 & 17 & 4.25 & 0 & 3934 & 231.4 \\
\hline
\end{tabular}

We made case studies on four test networks and the US-network $[11,16]$ which main characteristics are described in Table 1. ARPTL calculations are based on the reference reliability data from [17, 18]. The ILP formulations of basic SCA and SFPP were solved by the software ILOG OPL studio 3.6.1 on a $2.4 \mathrm{GHz}$ CPU computer running Windows 
2000 with 2 MB RAM within half an hour. The SFPP formulation has more restrictions than those of DFMR and leads to a higher computational effort.

\subsection{Experiment 1 with a goal to check $R_{2}$ benefit from SFPP}

Both Tables 2 and 3 compare the average $R_{2}$ of four test networks from two formulations of span restoration, i.e. basic SCA and SFPP, and show the $R_{2}$ enhancements from the solutions of SFPP compared with basic SCA. As described in Section 2.3, SFPP uses the minimum cost of a network obtained from basic SCA as the budget and purely optimizes the routing. The goal of this experiment is to find how much $R_{2}$ benefit can be received due to the optimization of SFPP. The computation for Table 2 uses the complete set of backup routes for each span without any limitations. In Table 3, the results of average $R_{2}$ under three different models (see the second, third and fourth columns) are all computed with the same spare capacity for each network. The minimum capacity of each test network was obtained from basic SCA with the limitation of only five backup routes available for each span. The column "SFPP (5 backups)" still uses 5 backup routes in the optimization. The column "SFPP (all backups)" exploits all backup routes for each span, but with the same spare capacity as "SFPP (5 backups)". The computation for the first case, "SFPP (5 backups)", becomes much easier. For the second case "SFPP (all backups)", it is computationally more difficult to find an optimized solution in a reasonably long time. Network $\mathrm{n} 8 \mathrm{~s} 17$ has a high average nodal degree and more candidate backup routes for each span, which leads to an unresolved problem to the computer. That's why the data of "SFPP (all backups)" for n8s17 network in Table 3 is not available.

When some restrictions on the number of backup routes are set, e.g. five backup routes for each span, $R_{2}$ can only be very limitedly enhanced, under $5 \%$ or even lower (see the last column of Table 3 ). If the complete set of backup routes for each span can be exploited, higher enhancement in $R_{2}$ can be achieved as shown in Table 2. The column "SFPP (all backups)" of Table 3 shows a big enhancement than its left column "SFPP (5 backups)". It is concluded that more backup routes can bring higher $R_{2}$ because of greater flexibility for traffic rerouting.

Table 2: $R_{2}$ enhancement from SFPP (all possible backup routes for each span are used)

\begin{tabular}{|l|l|l|c|}
\hline \multirow{2}{*}{ Test networks } & \multicolumn{2}{|c|}{ Average $R_{2}$} & \multirow{2}{*}{$\begin{array}{c}R_{2} \text { enhancement } \\
(\%)\end{array}$} \\
\cline { 2 - 3 } & Basic SCA & SFPP & 6 \\
\hline $\mathrm{n} 11 \mathrm{~s} 17$ & 0.538 & 0.570 & 14.3 \\
\hline $\mathrm{n} 9 \mathrm{~s} 15$ & 0.559 & 0.639 & 5.5 \\
\hline $\mathrm{n} 11 \mathrm{~s} 20$ & 0.640 & 0.675 & 3.7 \\
\hline $\mathrm{n} 8 \mathrm{~s} 17$ & 0.587 & 0.609 & \\
\hline
\end{tabular}

Table 3: $R_{2}$ enhancement from SFPP (Basic SCA provides the spare capacity with 5 backup routes for each span)

\begin{tabular}{|c|c|c|c|c|}
\hline \multirow{2}{*}{$\begin{array}{l}\text { Test } \\
\text { networks }\end{array}$} & \multicolumn{3}{|c|}{ Average $R_{2}$} & $\begin{array}{c}R_{2} \text { enhancement } \\
\text { (5 backups) (\%) }\end{array}$ \\
\cline { 2 - 4 } & $\begin{array}{l}\text { Basic SCA } \\
\text { (5 backups) }\end{array}$ & $\begin{array}{l}\text { SFPP } \\
\text { (5 backups) }\end{array}$ & $\begin{array}{c}\text { SFPP } \\
\text { (all backups) }\end{array}$ & \\
\hline $\mathrm{n} 11 \mathrm{~s} 17$ & 0.655 & 0.659 & 0.702 & 0.6 \\
\hline $\mathrm{n} 9 \mathrm{~s} 15$ & 0.639 & 0.671 & 0.688 & 5.1 \\
\hline $\mathrm{n} 11 \mathrm{~s} 20$ & 0.752 & 0.760 & 0.772 & 1.1 \\
\hline $\mathrm{n} 8 \mathrm{~s} 17$ & 0.714 & 0.740 & $\mathrm{n} / \mathrm{a}$ & 3.7 \\
\hline
\end{tabular}

\subsection{Experiment 2 with a goal to find the factors which affect path unavailability}

In this experiment, we do not make a traditional analysis on the physical factors, like the failure rates of optical cables and optical amplifiers, or any repair rates. However, we check the different factors affecting $R_{2}$ and path unavailability from the network design point of view.

By providing at most 5, 10, 20,30, 40, 50, 60 and 69 backup routes for each span, respectively, we made eight computations for the US network with span restoration in order to analyze the variation of network redundancy and $R_{2}$. The minimum cost with the particular set of backup routes is obtained by solving the formulation of basic SCA. If more than 69 backup routes are required, not every span can obtain the same number of backup routes due to the characteristics of the US network topology. The general tendency of Figure 1 shows that the network redundancy decreases with more available backup routes for each span. Here network redundancy is the ratio of the whole network spare capacity to the total working capacity. In other words, less investment is required to achieve full restorability to 
single span failures if more backup routes can be exploited. We notice that the network redundancy with 5 backup routes is obviously higher than the rest values when more backup routes are used. The network redundancies with 20,30 and 40 backup routes are identical. The values with 50,60,69 and even the complete set of backup routes (which isn't given in Figure 1) are identical as well. This illustrates that the necessary spare capacity remains the same within a certain number of backup routes and will not decrease continuously above a threshold number of routes. That means we cannot keep reducing necessary spare capacity by just exploiting more backup routes.

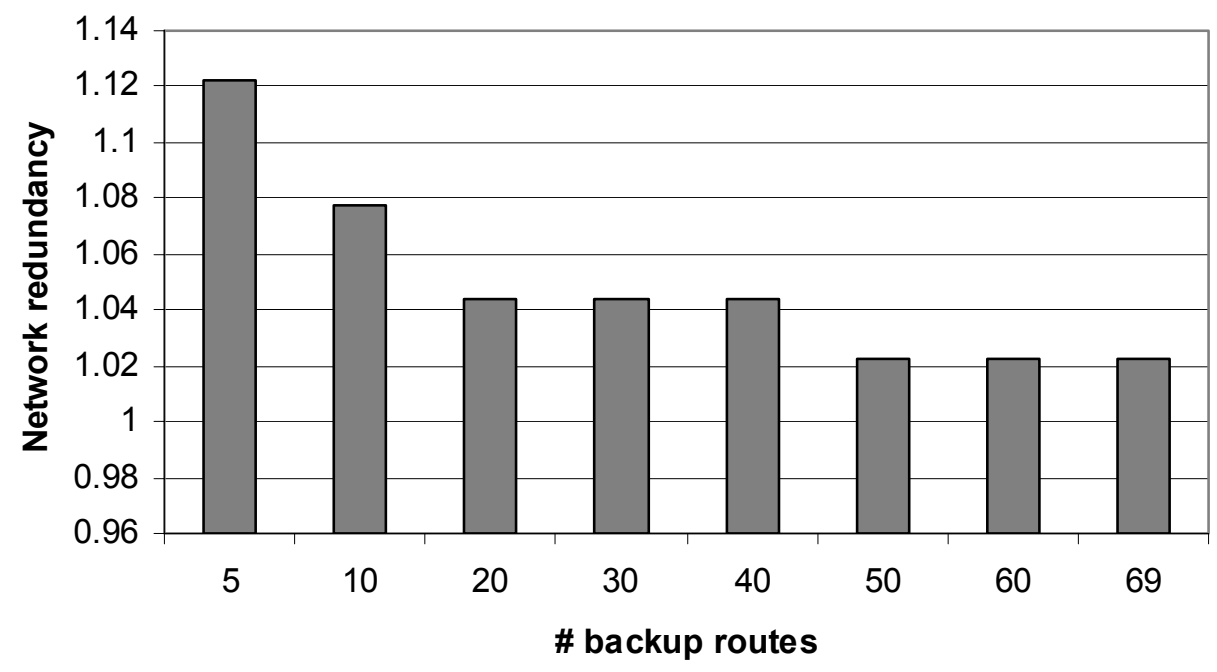

Figure 1: Network redundancy decreases with more available backup routes for each span in US network with span restoration.

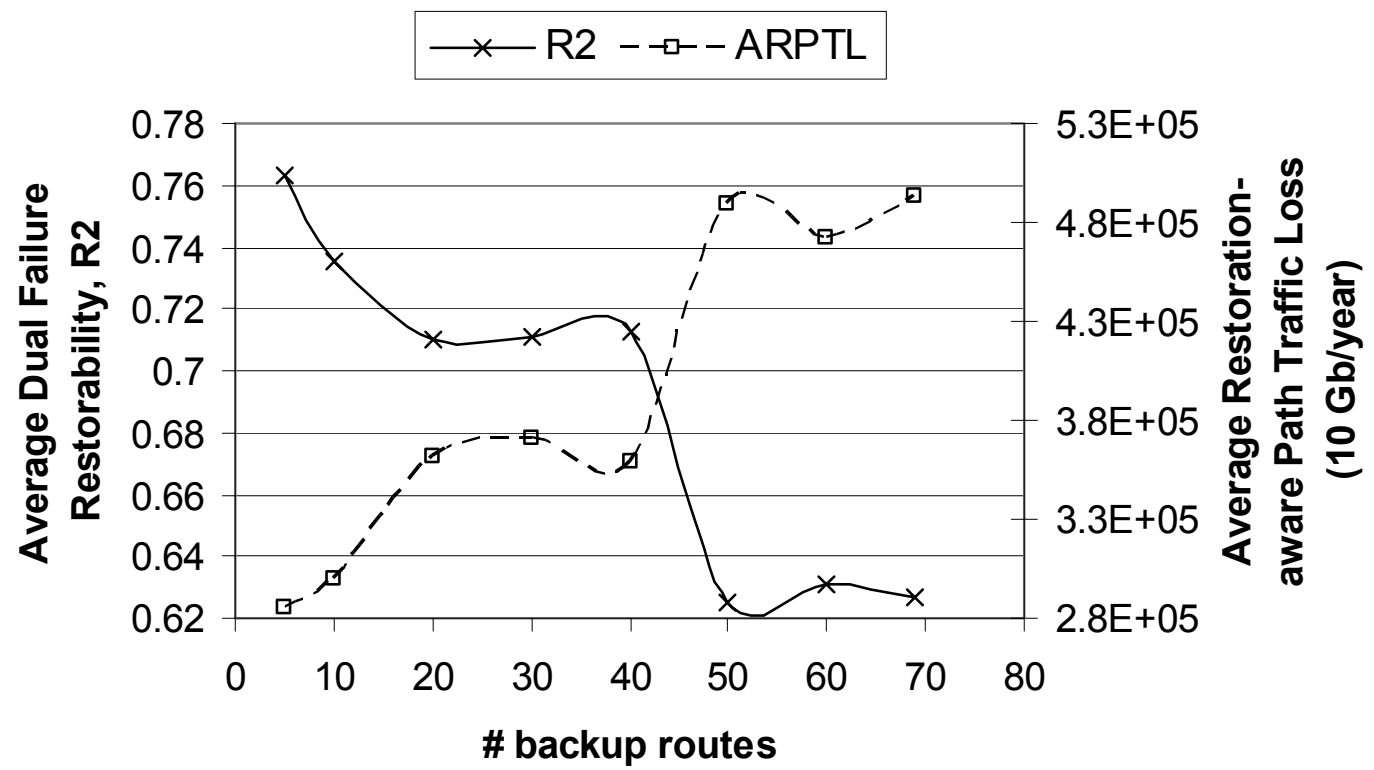

Figure 2: Variation of $R_{2}$ and ARPTL with the number of backup routes for each span in US network with span restoration. 
$R_{2}$ and ARPTL were then computed with different number of backup routes. In Figure 2 the solid curve connecting these data just shows the trend of $R_{2}$. The distributions of $R_{2}$ in Figure 2 and network redundancy in Figure 1 show a similar tendency in relation to the number of backup-routes. The higher the network redundancy (or the more the spare capacity), the higher $R_{2}$ becomes. If the redundancy does not change (e.g. the redundancies with 20, 30, 40 backup routes), the value of $R_{2}$ also remains almost the same based on the results from basic SCA. Certainly, as mentioned in Section 3.1, the optimization with more backup routes can reach a higher $R_{2}$ even if the same spare capacity is given. There is symmetry in the curves of $R_{2}$ and ARPTL. This shows that $R_{2}$ is an important factor for the path unavailability, here represented by average restoration aware path traffic loss ARPTL. The higher $R_{2}$, the higher path availability or the lower path unavailability becomes. Figure 3 depicts $R_{2}$ and ARPTL vary with network redundancies. Again, the symmetry with $R_{2}$ and ARPTL appears.

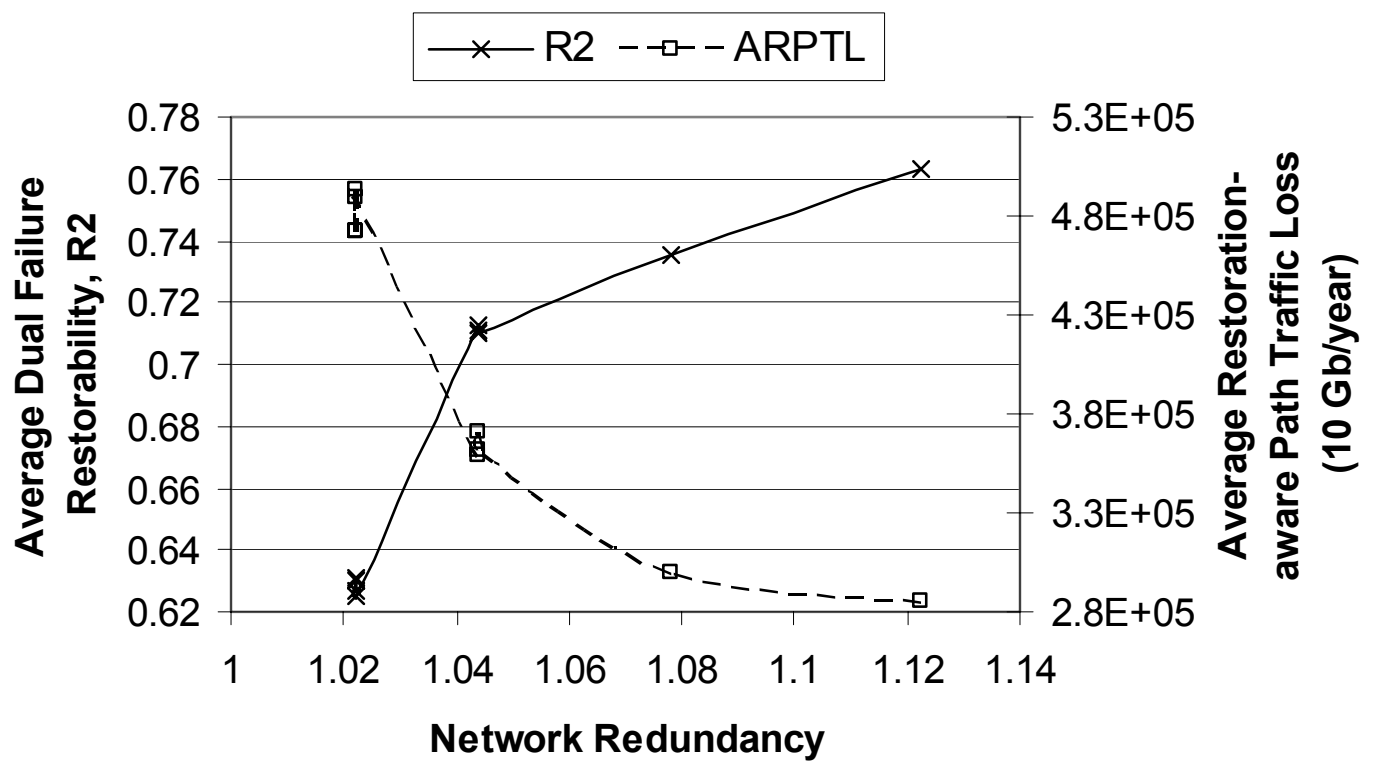

Figure 3: Variation of $R_{2}$ and ARPTL with network redundancy in US network under span restoration.

\section{CONCLUSIONS AND OUTLOOK}

In this work, a new concept of path unavailability is presented to help analysing the case of span restoration with shared spare capacity. An ILP formulation applied to span-restorable networks is introduced to maximize the dual-failure restorability with a given total cost restriction. This formulation, suitable for static pre-plans of span restoration, takes into account the failure sequence of the two failed spans and thus can guide network operator to implement the design explicitly. Various factors affecting path availability, like network redundancy, the number of backup routes for each span, and dual-failure restorability, are discussed from the standpoint of network design.

Five networks are investigated by applying the new "path unavailability" concept and the new formulation. From the experiments, we conclude the following:

1. The introduced formulation of SFPP reroutes the restoration paths optimally and achieves the maximum dual failure restorability under a static preplan with a given spare capacity. If the number of backup routes for each span is very limited, $R_{2}$ cannot benefit much from this formulation. When more backup routes are exploited, higher $R_{2}$ can be obtained through SFPP even if the same spare capacity is given.

2. Minimum spare capacity, obtained from the formulation of basic spare-capacity allocation (basic SCA), decreases with more backup routes for each span. However, we cannot keep reducing the spare capacity above a certain threshold. 
3. For the same network with span restoration, the higher network redundancy (or the more spare capacity) can expect the higher dual-span failure restorability.

4. $R_{2}$ is an important affecting factor for the path availability. The higher $R_{2}$, the higher path availability or the lower path unavailability.

Further investigation about path availability applied to other restoration schemes will be implemented. More formulations to enhance $R_{2}$ will be studied with different conditions.

\section{ACKNOWLEDGEMENT}

This work was partly financed by the Swiss Federal Office for Education and Science BBW (project C01.0087).

\section{REFERENCES}

1. M. Willebeek-LeMair and P.Shahabuddin. "Approximating dependability measures of computer networks: An fddi case study", IEEE/ACM Transactions on Networking, 5(2):311-327, April 1997.

2. M. Clouqueur, W. D. Grover, "Availability analysis of span-restorable mesh networks", IEEE journal on selected areas in communications, vol. 20, no. 4, May, 2002.

3. C. Assi, W. Huo, A. Shami, "Impact of Resource Sharability on Dual Failure Restorability in Optical Mesh Networks", IFIP NETWORKING 2005, Waterloo, Canada, May 2005.

4. C. Assi, W. Huo, A. Shami, N. Ghani, "Analysis of Capacity Re-Provisioning in Optical Mesh Networks", IEEE Communications Letters, vol. 9, no. 7, pp. 658-660, July 2005.

5. D. Griffith, K. Sriram, S. Lee, S. Klink, and N. Golmie, "Restorability versus Efficiency in $(1: 1)^{\mathrm{n}}$ Protection Schemes for Optical Networks", ICC 2004 Optical Networking Symposium.

6. D. Griffith, K. Sriram, S. Klink, and N. Golmie, "Optimal Mixtures of Different Types of Recovery Schemes in Optical Networks", Broadband Networks 2004-Broadband Optical Networking Symposium.

7. M. T. Frederick, P. Datta and A. K. Somani, "Evaluating Dual-Failure Restorability in Mesh-Restorable WDM Optical Networks", IEEE ICCCN2004, Oct. 2004.

8. M. Clouqueur, W. D. Grover, "Mesh-Restorable Networks with Enhanced Dual-Failure Restorability Properties", Photonic Network Communications, 9:1, 7-18, 2005.

9. W. D. Grover, Mesh-Based Survivable Networks, Options and Strategies for Optical, MPLS, SONET, and ATM Networking, New Jersey: Prentice Hall PTR, 2004.

10. A. Birolini. Reliability Engineering Theory and Practice. Berlin: Springer-Verlag, 1999.

11. L. Zhou, M. Held, L. Wosinska, "Analysis and optimization of connection availabilities in optical networks with different protection strategies", in Reliability of Optical Fibre Components, Devices, Systems, and Network II, Proc. SPIE Photonics Europe, Strasbourg, France, Apr.2004.

12. ITU-T Draft new Recommendation G.697, "Optical monitoring for DWDM systems", June 2004.

13. D. C. Kilper, R. Bach, D. J. Blumenthal, D. Einstein, T. Landolsi, L. Ostar, M. Preiss and A. E. Willner, "Optical Performance Monitoring”, in IEEE Journal of Lightwave Technology, 22 (1), pp. 294-304, January 2004.

14. M. Held, L. Wosinska, P. M. Nellen and C. Mauz, "Consideration of connection availability optimization in optical networks", DRCN03, Banff, Alberta, Canada, 2003.

15. M. Herzberg, S. Bye, "An optimal spare-capacity assignment model for survival networks with hops limits", in Proceedings of IEEE GLOBECOM 1994, San Francisco, CA, USA, November 1994, pp. 1601 - 1607.

16. R.Rawaswami, K.N. Sivarajan, Optical networks, a practical perspective, Morgan Kaufmann Publishers, $2^{\text {nd }}$ Edition, pp 667-696, 2002.

17. Mikac, B., R. Inkret, "Availability Model of WDM Optical Networks", Proceedings of the Int. Workshop on the Design of Reliable Communication Networks, DRCN 2000, April 9-12, 2000, Munich, Germany.

18. L. Wosinska and L. Pedersen, "Scalability limitations of optical networks due to reliability constraints", in Proceedings of NFOEC'01, Baltimore, July 2001. 